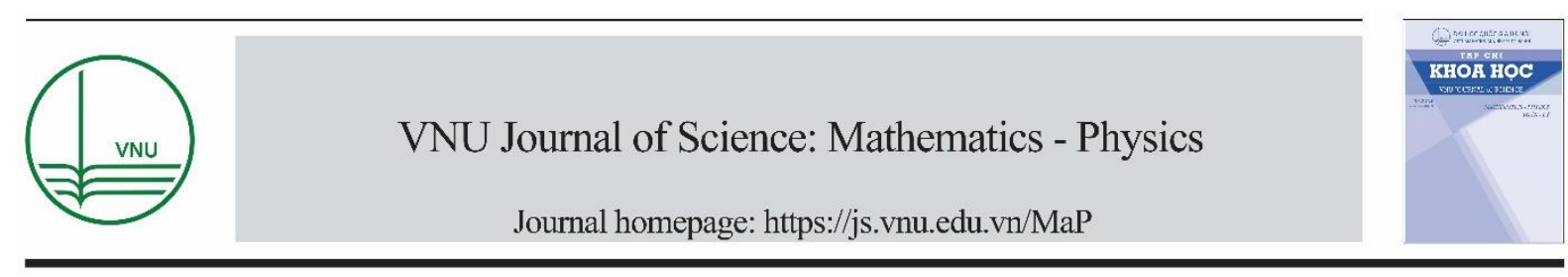

Original Article

\title{
Size Effect in Grand-canonical Monte-Carlo Simulation of Solutions of Electrolyte
}

\author{
Nguyen Viet Duc, ${ }^{2, *}$, Nguyen The Toan ${ }^{1,2}$ \\ ${ }^{I}$ VNU Key Laboratory of Multiscale Simulation of Complex Systems \\ ${ }^{2}$ Faculty of Physics, VNU University of Science, 334 Nguyen Trai, Thanh Xuan, Hanoi, Vietnam
}

Received 04 March 2019

Revised 06 May 2019; Accepted 15 May 2019

\begin{abstract}
A Grand-canonical Monte-Carlo simulation method is investigated. Due to charge neutrality requirement of electrolyte solutions, ions must be added to or removed from the system in groups. It is then implemented to simulate solution of 1:1, 2:1 and 2:2 salts at different concentrations using the primitive ion model. We investigate how the finite size of the simulation box can influence statistical quantities of the salt system. Remarkably, the method works well down to a system as small as one salt molecule. Although the fluctuation in the statistical quantities increases as the system gets smaller, their average values remain equal to their bulk value within the uncertainty error. Based on this knowledge, the osmotic pressures of the electrolyte solutions are calculated and shown to depend linearly on the salt concentrations within the concentration range simulated. Chemical potential of ionic salt that can be used for simulation of these salts in more complex system are calculated.
\end{abstract}

Keywords: GCMC, electrolyte solution simulation, primitive ion model, finite size effect.

\section{Introduction}

Computer simulation is an integral part of many areas of modern interdisciplinary research in physics, chemistry, biology and material science [1]. This is especially true for computer simulation of biological systems in medicine such as drug design and bioinspired novel materials and nanotechnology for medicine [2]. For such systems, molecular dynamics has been an important computational tool to understand physical characteristics of ligand receptor binding processes, and to predict structural, dynamical and thermodynamic properties of biological molecules. However, although computing

\footnotetext{
${ }^{*}$ Corresponding author.

Email address: ducnv84@gmail.com
}

https//doi.org/ 10.25073/2588-1124/vnumap.4296 
hardware has been steadily improved over the year, the large amount of atoms (correspondingly, the number of degrees of freedoms) in such system has rendered traditional molecular dynamics simulation to limited applications within few hundred nanoseconds and tens of nanometer scales. This computing requirement is even more demanding and challenging when the physics phenomenon involved require quantum mechanical simulation. To overcome such limitation and to bridge to larger time and spatial scales, multiscale simulation strategies have been an active research. Among them, methods of hybrid Quantum mechanics/Molecular mechanics or Coarse-grained/Molecular Mechanics simulation, or Adaptive resolution simulation have been proposed with limited success $[3,4,5,6,7]$. The general idea behind multiscale simulation is to focus in molecular details to only a small, well-defined region (MM region) of interest while the rest of the system can be simulated at a coarser scale, making the computation more efficient. The bridging of macro- molecules (such as protein or DNA) between two different scaled regions can be handled adequately in such hybrid simulation with suitable choice of coarse-grained model such as the Gö model $[8,9]$ for protein or similar coarse-grained model for DNA [10]. This multiscale strategy also helps to avoid unnecessary bias due to potentially wrong orientations of the side chains far from the binding site. However, the simulation of mobile molecules, especially mobile ions, into and out of the MM region is still an open question which is not trivial to handle in a molecular dynamic simulation. In fact, one usually forbids the mobile ions to move in and out of the MM region in such simulation. One idea to overcome this is to look beyond molecular dynamics. Specifically, in addition to molecular dynamics simulation, one could try to implement a Monte-Carlo simulation in the Grand canonical ensemble. In such simulation, mobile ions could be inserted and removed from the MM region in such a way that their chemical potentials are fixed, and controlled by coupling to a particle reservoir with the correct concentration. This is actually desirable because all biological systems function in equilibrium with water solutions at given $\mathrm{pH}$ and salinity. Of course, developing and implementing such scheme for application in computational biomedicine or pharmaceutical nanotechnology require large amount of time and resources and it is a very active research area.

In this paper, as a first step in such direction, we present a Grand canonical Monte-Carlo (GCMC) simulation of electrolyte solutions for different salinity expanding upon a preliminary study [11]. The Grand-Canonical Monte-Carlo method was developed and used in several recent papers in our group to study the condensation of DNA inside bacteriophages in the presence of mixture of different salts, $\mathrm{MgSO}_{4}, \mathrm{MgCl}_{2}, \mathrm{NaCl}[12,13,14,15]$. However, detail of the method was never presented, only the simulation results of DNA system were shown. In this paper, the methodology and implementation of this GCMC method is presented systematically and in detail. This allows for extension to any systems, not just DNA systems, and for potential integration in various multiscale simulation schemes.

The paper is organized as follows. In Sec. 2, the theory of Grand-canonical Monte-Carlo method is reviewed. In Sec. 3, the detail implementation of this method for various salts and the finite size effect are presented. Result for the fugacities and osmotic pressure are reported and discussed. We conclude in Sec. 4.

\section{Review of the theory of grand canonical Monte-Carlo simulation of electrolyte solutions}

In a Grand Canonical Monte-Carlo (GCMC) simulation, the number of ions is not constant during the simulation. Instead their chemical potentials are fixed. To show how this is done, let us consider a state $\mathrm{i}$ of the system that is characterized by the locations of $N_{i Z+}$ multivalent counterions, $N_{i+}$ monovalent counterions, $N_{i Z-}$ multivalent counterions, $N_{i-}$ coions. In the grand canonical ensemble of unlabeled particles, the probability of such state is given by: 


$$
\pi_{i}=\frac{1}{Z} \frac{1}{\Lambda_{Z+}^{3 N_{i} Z_{+}} \Lambda_{+}^{3 N_{i_{-}}} \Lambda_{Z-}^{3 N_{i} Z_{-}} \Lambda_{-}^{3 N_{i_{-}}}} \exp \left[\beta\left(\mu_{Z+} N_{i Z+}+\mu_{+} N_{i+}+\mu_{Z_{-}} N_{i z_{-}}+\mu_{-} N_{i_{-}}-U_{i}\right)\right]
$$

Here, $Z$ is the grand canonical partition function, $\beta=1 / k_{B} T, \Lambda_{x} \equiv h / \sqrt{2 \pi m_{x} k_{B} T}$ are the thermal wavelength of the corresponding ion type (here $x$ are either $Z+, Z-,-$ or + ), $U_{i}$ is the interaction energy of the state $i$, and $\mu_{x}$ are the corresponding chemical potential of the ions. In a standard Monte Carlo simulation, one would like to generate a Markov chain of system states i with a limiting probability distribution proportional to $\pi_{i}$. To do this, given a state $i$, one tries to move to state $j$ with probability $p_{i j}$. A sufficient condition for the Markov chain to have the correct limiting distribution is:

$$
\frac{p_{i j}}{p_{j i}}=\frac{\pi_{i}}{\pi_{j}}
$$

As usual, at each step of the chain, a "trial" move to change the system from state $i$ to state $j$ is attempted with probability $q_{i j}$ and is accepted with probability $f_{i j}$. Clearly,

$$
p_{i j}=q_{i j} f_{i j}
$$

It is convenient to regard the simulation box as consisting of $V$ discrete sites ( $V$ is very large). Then for a trial move where $v_{\alpha}$ particles of species $\alpha$ are added to the system

Conversely, if $v_{\alpha}$ particles of species $\alpha$ are removed from the system:

$$
\begin{gathered}
q_{i j}=\frac{1}{V^{v_{\alpha}} v_{\alpha} !} \\
q_{i j}=\frac{\left(N_{\alpha}-v_{\alpha}\right) !}{N_{\alpha} ! v_{\alpha}}
\end{gathered}
$$

Putting everything together, equations (1)-(5) give us a recipe to calculate the Metropolis acceptance probability of a particle insertion/deletion move in GCMC simulation. For example, if in a transition from state $i$ to state $j$, a multivalent salt molecule (one $Z$-ion and $Z$ coions) is added to the system, the Metropolis probability of acceptance of such move can be chosen as:

$$
f_{M}=\min \left\{1, f_{i j} / f_{j i}\right\}
$$

where

$$
\frac{f_{i j}}{f_{j i}}=\frac{B_{Z: 1}}{\left(N_{i Z+}+1\right)\left(N_{i-}+1\right) \ldots\left(N_{i-}+Z\right)} \exp \left[\beta\left(U_{i}-U_{j}\right)\right]
$$

with

$$
B_{Z: 1}=\exp \left(\beta \mu_{Z: 1}\right) \frac{V^{Z+1}}{\Lambda_{Z+}^{3} \Lambda_{-}^{3 Z}}
$$

and $\mu_{Z: 1}=\mu_{Z+}+Z \mu_{-}$is the combined chemical potential of a $Z: 1$ salt molecule. On the other hand, if a multivalent salt molecule (one $Z$-ion and $Z$ coions) is removed from the system, we have:

$$
\frac{f_{i j}}{f_{j i}}=\frac{N_{i Z+} N_{i-} \cdots\left(N_{i-}-Z+1\right)}{B_{Z: 1}} \exp \left[\beta\left(U_{i}-U_{j}\right)\right]
$$

Similar expressions are easily obtained from addition/removal of $Z: Z$ salt. For addition, 


$$
\frac{f_{i j}}{f_{j i}}=\frac{B_{Z: Z}}{\left(N_{i Z+}+1\right)\left(N_{i Z-}+1\right)} \exp \left[\beta\left(U_{i}-U_{j}\right)\right],
$$

and for removal,

$$
\frac{f_{i j}}{f_{j i}}=\frac{\left(N_{i Z+}+1\right)\left(N_{i Z-}+1\right)}{B_{Z: Z}} \exp \left[\beta\left(U_{i}-U_{j}\right)\right],
$$

where

$$
B_{Z: Z}=\exp \left(\beta \mu_{Z: Z}\right) \frac{V^{2}}{\Lambda_{\mathrm{Z}^{+}}^{3} \Lambda_{Z-}^{3}},
$$

and $\mu_{Z: Z}=\mu_{Z+}+\mu_{Z-}$ is the combined chemical potential of $Z: Z$ salt molecule. For the addition of monovalent 1: 1 salt to the system

$$
\frac{f_{i j}}{f_{j i}}=\frac{B_{1: 1}}{\left(N_{i+}+1\right)\left(N_{i-}+1\right)} \exp \left[\beta\left(U_{i}-U_{j}\right)\right],
$$

and for removal of $1: 1$ salt,

$$
\frac{f_{i j}}{f_{j i}}=\frac{N_{i+} N_{i-}}{B_{1: 1}} \exp \left[\beta\left(U_{i}-U_{j}\right)\right],
$$

where

$$
B_{1: 1}=\exp \left(\beta \mu_{1: 1}\right) \frac{V^{2}}{\Lambda_{+}^{3} \Lambda_{-}^{3}},
$$

and $\mu_{1: 1}=\mu_{+}+\mu_{-}$is the combined chemical potential of $1: 1$ salt molecule.

Beside particle addition/deletion moves, one also tries standard particle translation moves. They are carried out exactly like in the case of a canonical Monte-Carlo simulation. In a "trial" move from state $i$ to state $j$, an ion is chosen at random and is moved to a random position in a volume element surrounding its original position. The standard Metropolis probability is used for the acceptance of such "trial" move:

$$
f_{M}=\min \left\{1, \exp \left[\beta\left(U_{i}-U_{j}\right)\right]\right\}
$$

\section{Grand canonical Monte-Carlo simulation of electrolyte solution in primitive ion model}

In this section, the application of the grand canonical Monte-Carlo simulation detailed in previous section to simulate a bulk concentration of electrolyte solution is presented. We will focus on the cases of 1:1,2:1 and 2:2 salt solution. For simplicity, all ions have radius of $\sigma_{x}=2 \AA$. The primitive ion model is used. The aqueous solution is modeled implicitly as a continuous medium with dielectric constant, $\varepsilon$. The interaction between two ions $\alpha$ and $\beta$ with radii $\sigma_{\alpha, \beta}$ and charges $Q_{\alpha, \beta}$ is given by

$$
U= \begin{cases}\frac{Q_{\alpha} Q_{\beta}}{\varepsilon r_{\alpha \beta}}, & \text { if } r_{\alpha \beta}>\sigma_{\alpha}+\sigma_{\beta} \\ \infty, & \text { if } r_{\alpha \beta}<\sigma_{\alpha}+\sigma_{\beta}\end{cases}
$$


Where $r_{\alpha \beta}=\left|\boldsymbol{r}_{\alpha}-\boldsymbol{r}_{\beta}\right| \quad$ is the distance between the ions. The simulation is carried out using the periodic boundary condition. The long-range electrostatic interactions between charges in neighboring cells are treated using the standard Ewald summation method [16]. To be able to calculate the pressure of the system, the Expanded Ensemble method [17, 18] is implemented. This method allows us to calculate the difference of the system free energies at different volumes by sampling these volumes simultaneously in a simulation run. By sampling two nearly equal volumes, $V$ and $+\Delta V$, and calculate the free energy difference $\Delta \Omega$, we can calculate the total pressure of the system:

$$
P\left(T, V,\left\{\mu_{x}\right\}\right)=-\left.\frac{\partial \Omega\left(T, V,\left\{\mu_{x}\right\}\right)}{\partial V}\right|_{T,\left\{\mu_{x}\right\}} \approx-\frac{\Delta \Omega}{\Delta V}
$$

The derivative of grand potential is taken with respect to volume at constant values of temperature and all four chemical potentials, $\left\{\mu_{x}\right\} \equiv\left\{\mu_{z_{+}}, \mu_{z_{-}}, \mu_{+}, \mu_{-}\right\}$. For each simulation run, 100 million MC moves are carried out depending on the average number of ions in the system. To ensure thermalization, 10 million initial moves are discarded before doing statistical analysis of the result of the simulation. All simulations are done using the physics simulation library SimEngine develop by one of the author (TTN). This library use OpenCL and OpenMP extensions of the C programming language to distribute computational workloads on multi-core CPU and GPGPU to speed up the simulation time. Both molecular dynamics and Monte-Carlo simulation methods are supported. In this paper the Monte-Carlo module of the library is used.

\section{A. Finite size effect}

The first question one asks is the limit of application of this GCMC method. For large system where the fluctuation in the particle number is fractionally small, the simulation result should give the same statistical property of canonical system. However, for small system where the particle number fluctuation is large, one might question of validity of the proposed method. To investigate this finite size effect, we simulate a salt solution at the same chemical potentials (resulting in the same expected salt concentrations), but with different volume dimensions. Specifically, the scaled fugacities are $B_{2: 2} / V^{2}=2.05 \times 10^{-10} \AA^{-2}, B_{2: 1} / V^{3}=1.14 \times 10^{-14} \AA^{-3}$ and $B_{1: 1} / V^{2}=5.50 \times 10^{-10} \AA^{-2}$. The simulation box is a cubic box with side length varying from $20 \AA$ to $120 \AA$, corresponds to the average number of particle of divalent anions from 0.7 to about 215.6. In Figure 1, the resultant concentrations at a given chemical potential is plotted as function of simulation box lengths. Similarly, Table 1 shows the numerical values obtained from our simulation for the averaged concentrations, particle numbers and osmotic pressures as function of the simulation box lengths. We can see that the GCMC method works very well down to a very small box size where the average number of particles is less than 1 . Indeed, within the uncertainty of the results, all concentrations are independent of simulation box length down to a very small box length. One only sees the finite size effect at simulation box length of about $30 \AA$ or smaller. At these small volumes, the average number of salt molecules in the simulation box is even smaller than one for some types (such as the number of +1 ions as shown in Table 1). This suggests that as long as the simulation box are large enough to have a few ions in it on average, the grand canonical Monte-Carlo method presented is reliable.

Our results show the reliability of our grand-canonical Monte-Carlo simulation method down to a system of as small as one salt particle. This is not only a result of our correct formulation and derivation of the method from the generating partition function. We believe it is also the result of the fact that the chemical potential contains mostly the self-energy contribution. Due to the periodic boundary condition 
employed, even when only one particle is present in the system, the electrostatic self-energy, on average, doesn't differ much from that of larger system.

Table 1. The result salt concentrations and osmotic pressure of the solution as function of the length of the cubic simulation box. The chemical potentials are fixed to have the desired mixture of concentrations of $200 \mathrm{mM}, 10 \mathrm{mM}$ and $50 \mathrm{mM}$ for 2:2 salt, 2:1 salt and 1:1 salt correspondingly.

\begin{tabular}{lllllll}
\hline $\begin{array}{l}\text { Box } \\
\text { length }(\AA)\end{array}$ & $c_{2: 2}(\mathrm{mM})$ & $c_{2: 1}(\mathrm{mM})$ & $c_{1: 1}(\mathrm{mM})$ & $N_{2+}$ & $N_{1+}$ & $P_{b}(\mathrm{~atm})$ \\
\hline 120 & $197.2 \pm 12.6$ & $10.0 \pm 42.7$ & $50.1 \pm 6.8$ & $215.60 \pm 13.16$ & $52.11 \pm 7.11$ & $8.66 \pm 0.20$ \\
100 & $197.0 \pm 16.7$ & $9.9 \pm 16.9$ & $50.2 \pm 8.9$ & $124.64 \pm 10.16$ & $30.21 \pm 5.37$ & $8.59 \pm 0.10$ \\
80 & $196.4 \pm 23.6$ & $10.1 \pm 24.1$ & $50.0 \pm 12.5$ & $63.67 \pm 7.44$ & $15.43 \pm 3.84$ & $8.73 \pm 0.15$ \\
60 & $197.6 \pm 37.2$ & $10.1 \pm 15.8$ & $50.0 \pm 19.2$ & $27.00 \pm 4.86$ & $6.51 \pm 2.50$ & $8.55 \pm 0.16$ \\
40 & $197.1 \pm 68.5$ & $9.9 \pm 68.9$ & $50.2 \pm 35.3$ & $7.98 \pm 2.65$ & $1.93 \pm 1.36$ & $8.76 \pm 0.04$ \\
30 & $193.9 \pm 104.7$ & $9.5 \pm 105.5$ & $48.0 \pm 54.9$ & $3.31 \pm 1.72$ & $0.78 \pm 0.89$ & $8.53 \pm 0.18$ \\
20 & $144.5 \pm 175.6$ & $3.3 \pm 178.1$ & $18.7 \pm 70.4$ & $0.71 \pm 0.86$ & $0.09 \pm 0.34$ & $3.84 \pm 0.10$ \\
\hline
\end{tabular}

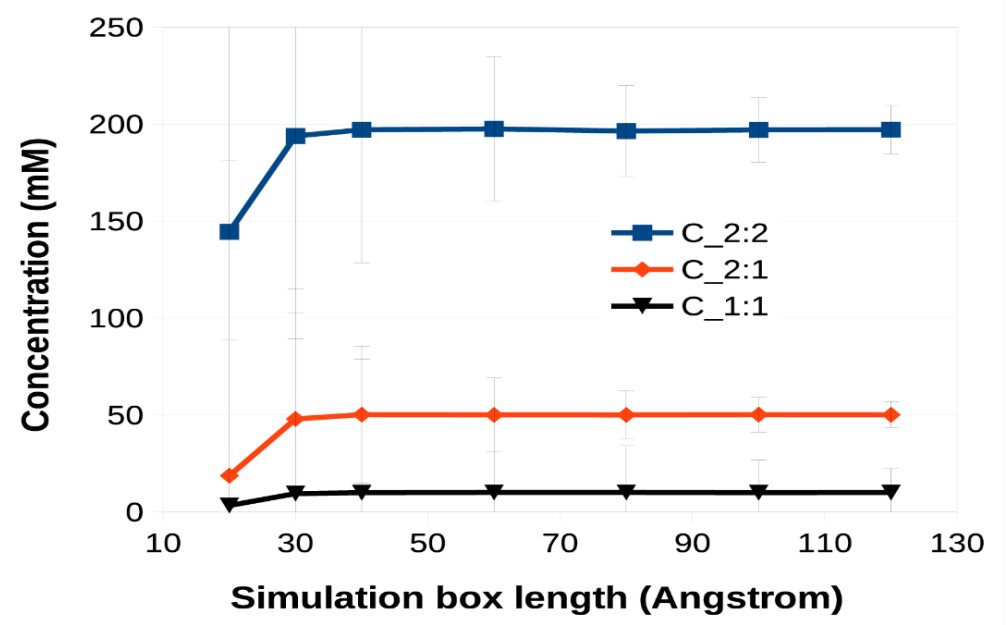

Fig 1. The concentrations of various component salt in a mixture of three different salts: 2:2, 2:1 and 1:1 salts. The chemical potentials of salt molecules are fixed. The size of the simulation box varies from $120^{\circ} \mathrm{A}$ down to 20A. Size dependent effect is only observed for very small simulation volume such that, on average, there is less than one salt particle in the volume.

For a given desired concentration, the chemical potential of the salts are independent on the sizes and shapes of the simulation box. It should be mentioned here the obvious effect of reducing the simulation box size is the increase in the relative fluctuation in concentrations. This is in line with statistical theory which says that the particle number fluctuation increases as $\sqrt{N}$ with the number of particle, $N$. The columns 5 and 6 of Table I clearly show this quantitative trend. Because of this, the number fluctuation increases relatively as $1 / \sqrt{N}$ as $N$ decreases. The error bar in Fig. 1 becomes very large at small simulation box size. Impressively, column 5 and 6 of Table I show that the $\sqrt{N}$ estimate for fluctuation in the number of particles works even for the case the average number of ions is smaller than one. In the rest of this paper, the simulation box volume is fixed $\mathrm{V}=2.650 \times 103 \mathrm{~nm}^{3}$, corresponding to a box length of $138.4 \AA$, more than enough to eliminate possible finite size effects even at some small salt concentrations simulated. 
Table 2. The scaled fugacity, B1:1 of the 1:1 salt at different concentrations. Columns 2 and 3 show the corresponding salt concentration and osmotic pressure of the salt bulk solution obtained from simulation.

\begin{tabular}{cll}
\hline$B_{1: 1} / V^{2}\left(\AA^{-2}\right)$ & $c(\mathrm{mM})$ & $P_{b}(\mathrm{~atm})$ \\
\hline $4.00 \times 10^{-11}$ & $11.7 \pm 1.9$ & $0.552 \pm 0.003$ \\
$1.15 \times 10^{-10}$ & $20.3 \pm 2.6$ & $0.954 \pm 0.007$ \\
$6.60 \times 10^{-10}$ & $51.99 \pm 4.2$ & $2.40 \pm 0.012$ \\
$2.30 \times 10^{-9}$ & $101.4 \pm 5.7$ & $4.683 \pm 0.023$ \\
$8.80 \times 10^{-9}$ & $206.2 \pm 10.2$ & $9.572 \pm 0.001$ \\
\hline
\end{tabular}

\section{B. Single salt solution}

Let us present the result of our GCMC simulations for solution containing a single type of salt, either 1:1, 2:1 or $2: 2$ salt. Some concentrations simulated are already performed independently by the authors of Ref. 11. For these concentrations, our results agree with their results. Thus, this section also serves as a check on the correctness of our code implementation. Tables II, III, and IV show the scaled fugacity $\mathrm{B}$ and the resultant averaged concentration of the solution obtained from simulation using these parameters. Three different salts, $1: 1$ salt, $2: 1$ salt and $2: 2$ salt are listed. Standard deviations in the concentration are about $10 \%$ in our simulation. This relative error is in line with those of previous GCMC simulations of Ref. 11. Additionally, the osmotic pressure of the solution obtained from simulation is presented in column 3. These values are also plotted in Fig. 2 for easier comparison. As one can see, at the same concentration, the osmotic pressure of 2:2 salt solution is lowest, while that of 2:1 salt is highest. This behavior can be understood. Figure 2 shows that, for the concentration range studied, the osmotic pressure increases linearly with concentration. At these low concentrations, our solution should follow the van der Waals equation of state [19]:

$$
\left(P+\frac{n^{2} a}{V^{2}}\right)(V-n b)=n R T
$$

where $n$ is the number of moles of the particles and $a$, and $b$ are the pressure and volume corrections due to non-ideality. The volume correction parameter, $b$, of this equation is

Table 3. The scaled fugacity, B2:1 of the 2:1 salt for different concentrations. Columns 2 and 3 show the corresponding salt concentration and osmotic pressure of the bulk salt solution obtained from simulation.

\begin{tabular}{lll}
\hline$B_{2: 1} / V^{3}\left(\AA^{-2}\right)$ & $\mathrm{c}(\mathrm{mM})$ & $P_{b}(\mathrm{~atm})$ \\
\hline $3.22 \times 10^{-16}$ & $10.03 \pm 1.56$ & $0.066 \pm 0.005$ \\
$1.80 \times 10^{-15}$ & $19.60 \pm 2.19$ & $1.26 \pm 0.008$ \\
$1.90 \times 10^{-14}$ & $50.75 \pm 3.69$ & $3.16 \pm 0.03$ \\
$1.00 \times 10^{-13}$ & $100.80 \pm 7.71$ & $6.16 \pm 0.05$ \\
$8.90 \times 10^{-13}$ & $245.57 \pm 9.63$ & $15.03 \pm 0.07$ \\
\hline
\end{tabular}

Table 4. The scaled fugacity, B2:2 of the 2:2 salt for different salt concentrations. Columns 2 and 3 show the corresponding salt concentration and osmotic pressure of the bulk salt solution obtained from simulation.

\begin{tabular}{lll}
\hline$B_{2: 2} / V^{2}\left(\AA^{-2}\right)$ & $\mathrm{c}(\mathrm{mM})$ & $P_{b}(\mathrm{~atm})$ \\
$6.36 \times 10^{-12}$ & $10.03 \pm 2.26$ & $0.379 \pm 0.003$ \\
$1.50 \times 10^{-11}$ & $20.81 \pm 3.07$ & $0.709 \pm 0.028$ \\
$4.45 \times 10^{-11}$ & $50.56 \pm 5.37$ & $1.60 \pm 0.016$ \\
$9.70 \times 10^{-11}$ & $100.81 \pm 7.29$ & $2.96 \pm 0.033$ \\
$2.50 \times 10^{-10}$ & $241.39 \pm 14.68$ & $6.82 \pm 0.130$ \\
\hline
\end{tabular}




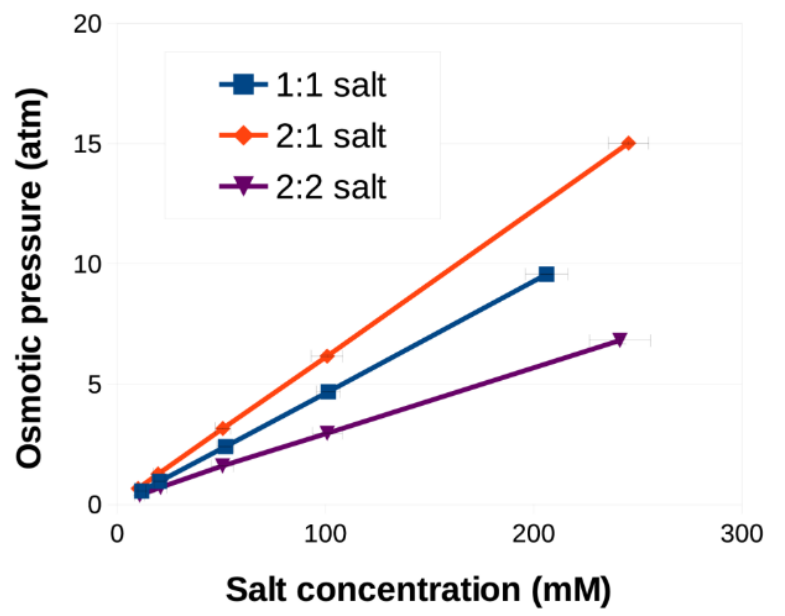

Fig. 2. The osmotic pressure of the electrolyte solution containing a single type of salt. The pressure increases linearly with concentration within the range studied

small for our system. However, the pressure correction parameter, $a$, of the van der Waals equation of state depends on interactions among different ions. This is why, at the same concentration, both 1:1 salt and 2:2 salt contain the same number of ions but the pressure of 2:2 salt solution is lower due to much stronger attraction among cations and anions. On the other hand, for 2:1 salt, there are 3 ions dissolved per molecule compared to 2 ions dissolved for the other two salts. As a result, the number of moles of particles are 1.5 times higher than other solution, $n_{2: 1}=1.5 n_{1: 1}$, leading to higher pressure.

\section{Conclusion}

In this paper, we presented an extensive study of the finite size effect on the Grand- Canonical Monte-Carlo simulation for electrolyte solutions using a primitive ion mode. It is shown that the method works remarkably well down to system as small as containing one salt molecule. Application of this method to simulate solutions containing single salt is carried out. The fugacities of individual salt species for different solutions at typical concentrations are reported. The result of osmotic pressure of the electrolyte solution are calculated and shown to be linearly proportional to the salt concentration within the range of concentrations considered. However, the pressure differs for different type of salt because the non-ideal gas corrections are different for different ion valence.

In this paper, the aqueous solution is simulated implicitly. It appears only in the dielectric constant of the medium. Our method is suitable therefore for a coarse-grained region in a multiscale simulation setup. If one simulates the solvent molecules explicitly, it is likely that a full particle insertion or deletion would be impractical due to a large change in the system energy. In such case, partial deletion/insertion of particle is preferable. Nevertheless, it is very unlikely one would practically need grand-canonical simulation in the atomistic region in a multiscale simulation.

\section{Acknowledgments}

We would like to thank Drs. T. X. Hoang and Paolo Carloni for valuable discussions. TTN acknowledges the financial support of the Vietnam National University grant number QG.16.01. The 
authors are indebted to Dr A. Lyubartsev for providing us with the Fortran source code of their Expanded Ensemble Method.

\section{References}

[1] M. P. Allen and D. J. Tildesley, Computer Simulation of Liquids, Clarendon Press, Oxford, 1987.

[2] P. Coveney, Computational biomedicine: modelling the human body, Oxford University Press, 2014.

[3] M. Praprotnik and L. D. Site, Multiscale Molecular Modeling, in: L. Monticelli and E. Salonen, Biomolecular Simulations: Methods and Protocols, Humana Press, Hatfield, Hertfordshire, 2013, ch. III, pp. 567-583.

[4] R. Potestio et al., Hamiltonian adaptive resolution simulation for molecular liquids, Phys. Rev. Lett. 110 (2013), 108301. https://doi.org/10.1103/PhysRevLett.110.108301.

[5] M. Neri, C. Anselmi, M. Cascella, A. Maritan, and A. Carloni, Coarse-Grained Model of Proteins Incorporating Atomistic Detail of the Active Site, Phys. Rev. Lett. 95 (2005), 218102. https://doi.org/10.1103/PhysRevLett.95.218102.

[6] W. G. Noid, Perspective: Coarse-grained models for biomolecular systems, J. Chem. Phys. 139 (2013), 090901. https://doi.org/10.1063/1.4818908.

[7] E. Brunk and U. Rothlisberger, Mixed Quantum Mechanical/Molecular Mechanical Molecular Dynamics Simulations of Biological Systems in Ground and Electronically Excited States, Chemical reviews 115 (2015), 6217-6263. https://doi.org/10.1021/cr500628b.

[8] Y. Ueda, H. Taketomi, and N. Go, Studies on Protein Folding, Unfolding, and Fluctuations by Computer Simulation. II. A Three-Dimensional Lattice Model of Lysozyme, Biopolymers 17 (1978), 15311548. https://doi.org/10.1002/bip.1978.360170612.

[9] T. X. Hoang and M. Cieplak, Molecular dynamics of folding of secondary structures in Go-type models of proteins, J. Chem. Phys. 112 (2000), 6851-6862. https://doi.org/10.1063/1.481261.

[10] E. Villa, A. Balaeff, and K. Schulten, Structural dynamics of the lac repressor-DNA complex revealed by a multiscale simulation, Proc. Nat. Acad. Science 102 (2005), 6783. https://doi.org/10.1073/pnas.0409387102.

[11] J. P. Valleau and L. K. Cohen, Primitive model electrolytes. I. Grand canonical Monte Carlo computations, J. Chem. Phys. 72 (1980), 5935-5941. https://doi.org/10.1063/1.439092.

[12] S. Lee, T. T. Le, and T. T. Nguyen, Reentrant Behavior of Divalent-Counterion-Mediated DNA-DNA Electrostatic Interaction, Phys. Rev. Lett. 105 (2010), 248101. https://doi.org/10.1103/PhysRevLett.105.248101.

[13] N. T. Toan, Strongly correlated electrostatics of viral genome packaging, J. Biol. Phys. 39 (2013), 247265. https://doi.org/10.1007/s10867-013-9301-4.

[14] T. T. Nguyen, Grand-canonical simulation of DNA condensation with two salts, effect of divalent counterion size, J. Chem. Phys. 144 (2016), 065102. https://doi.org/10.1063/1.4940312.

[15] V. D. Nguyen, T. T. Nguyen, and P. Carloni, DNA like-charge attraction and overcharging by divalent counterions in the presence of divalent co-ions, J. Biol. Phys. 43 (2017), 185-195. https://doi.org/10.1007/s10867-017-9443-x.

[16] P. P. Ewald, Evaluation of optical and electrostatic lattice potentials, Ann. Phys. 64 (1921), 253.

[17] L. Nordenskiöld and A. P. Lyubartsev, Monte Carlo Simulation Study of Ion Distribution and Osmotic Pressure in Hexagonally Oriented DNA, J. Phys. Chem. 99 (1995), 10373-10382. https://doi.org/10.1021/j100025a046.

[18] Lars Guldbrand, Lars G. Nilsson, and Lars Nordenskiöld, A Monte Carlo simulation study of electrostatic forces between hexagonally packed DNA double helices, J. Chem. Phys. 85 (1986), 6686 6698. https://doi.org/10.1063/1.451450.

[19] L. Landau and E. Lifshitz, Statistical Physics, Elsevier Science, 2013. 